\title{
The Spin Period, Luminosity, and Age Distributions of Anomalous X\#Ray Pulsars
}

\section{Citation}

Chatterjee, Pinaki, and Lars Hernquist. 2000. "The Spin Period, Luminosity, and Age

Distributions of Anomalous X\#Ray Pulsars." The Astrophysical Journal 543 (1): 368-72. https:// doi.org/10.1086/317097.

\section{Permanent link}

http://nrs.harvard.edu/urn-3:HUL.InstRepos:41381790

\section{Terms of Use}

This article was downloaded from Harvard University's DASH repository, and is made available under the terms and conditions applicable to Other Posted Material, as set forth at http:// nrs.harvard.edu/urn-3:HUL.InstRepos:dash.current.terms-of-use\#LAA

\section{Share Your Story}

The Harvard community has made this article openly available.

Please share how this access benefits you. Submit a story. 


\title{
The Spin Period, Luminosity and Age Distributions of Anomalous X-Ray Pulsars
}

\author{
Pinaki Chatterjee ${ }^{1} \&$ Lars Hernquist ${ }^{2}$ \\ Harvard-Smithsonian Center for Astrophysics, 60 Garden Street, Cambridge, MA 02138
}

\begin{abstract}
We consider the accretion model for anomalous X-ray pulsars proposed recently by Chatterjee, Hernquist and Narayan, in which the emission is powered by accretion from a fossil disk formed by the fallback of material from a supernova explosion. We demonstrate that this model is able to account for the spin period, luminosity and age distributions of the observed population of AXPs for reasonable and broad distributions of the free parameters of the model, namely, the surface magnetic field of the neutron star, the mass of its accretion disk and its initial spin period. In particular, this model is able statistically to account for the puzzlingly narrow observed spin distribution of the AXPs. We show also that if the establishment of fallback accretion disks around isolated neutron stars is a universal phenomenon, then a fairly large minority $(\sim 20 \%)$ of these objects become X-ray bright AXPs or X-ray faint systems spinning down by propeller action, while the rest become radio pulsars.
\end{abstract}

Subject headings: stars: neutron - pulsars: general - accretion, accretion disks X-rays: stars

\section{Introduction}

Anomalous X-ray pulsars (AXPs), about half a dozen of which are known, have properties significantly different from those of binary X-ray pulsars (see Mereghetti 1999 for a recent review). AXPs are sources of pulsed X-ray emission with relatively low persistent $\mathrm{X}$-ray luminosities, $L_{x} \sim 10^{35}-10^{36} \mathrm{erg} / \mathrm{sec}$, and soft spectra which are well-fitted by a combination of blackbody and power-law contributions, with effective temperatures and

\footnotetext{
${ }^{1}$ pchatterjee@cfa.harvard.edu

${ }^{2}$ lars@cfa.harvard.edu
} 
photon indices in the range $T_{e} \sim 0.3-0.4 \mathrm{keV}$ and $\Gamma \sim 3-4$, respectively. They have relatively long spin periods of about $P \sim 6-12$ seconds, which increase steadily with time. Their characteristic ages are about $P / 2 \dot{P} \sim 10^{3}-10^{5}$ years. No binary companions have been detected for these objects, and observations have placed strong constraints on companion masses (e.g. Mereghetti, Israel \& Stella 1998; Wilson et al. 1998). At least three AXPs have been associated with young supernova remnants which limit their ages to $\sim 10-20 \mathrm{kyr}$.

There are at present two theories seeking to explain the properties of AXPs. In one, they are modeled as isolated, ultramagnetized neutron stars or "magnetars" spinning down by the emission of magnetic dipole radiation, which is powered either by residual thermal energy (Heyl \& Hernquist 1997a,b) or by magnetic field decay (Thompson \& Duncan 1996). The required surface magnetic field strength is $B \sim 10^{14}-10^{15} \mathrm{G}$, which is similar to the values inferred from timing data for soft gamma repeaters or SGRs (e.g. Kouveliotou et al. 1998, 1999; see, however, Marsden, Rothschild \& Lingenfelter 1999).

The other class of theories proposes that the X-ray emission from AXPs is powered by accretion from binary companions of very low mass (Mereghetti \& Stella 1995), from the interstellar medium (see Wang 1997 in reference to the AXP candidate RX J0720.4-3125; however, see Heyl \& Hernquist 1998 for a magnetar model of this object), or from the debris of a disrupted high-mass X-ray binary system, after a stage of common-envelope evolution (van Paradijs et al. 1995; Ghosh, Angelini \& White 1997). The inferred magnetic field strengths of the neutron stars then are similar to those of ordinary radio pulsars and luminous X-ray pulsars $\left(B \lesssim 10^{12} \mathrm{G}\right)$.

More recently, Chatterjee, Hernquist and Narayan (1999; hereafter CHN) proposed another accretion model in which AXPs are neutron stars with standard magnetic fields accreting from a disk formed after fallback of material from a supernova explosion. The star is rapidly spun down close to the observed AXP periods on a timescale of $\sim 10^{4}$ years by the action of a propeller effect (for other accretion scenarios for AXPs and SGRs based on the propeller effect, see Alpar [1999] and Marsden et al. [1999]).

However, it has been observed that the spin-down of certain AXPs is very stable over time (Kaspi, Chakrabarty \& Steinberger 1999). This could pose a problem for all accretion models, in which a higher level of timing noise might be expected. Moreover, it is not clear whether optical and infra-red emission from such accretion disks would be low enough to evade the limits set by observations (Perna, Hernquist \& Narayan, 1999; Hulleman et al. 2000). It remains to be seen whether accretion models can circumvent these difficulties.

A question that has hitherto largely been unaddressed is the reason for the puzzlingly 
strong clustering of spin periods of the observed AXPs. Colpi, Geppert \& Page (2000) contend that this phenomenon implies magnetic field decay in the magnetar model. Marsden et al. (1999) seek to explain this by limiting the magnetic fields of the neutron stars and the strength of the propeller wind emission by which they spin down to narrow ranges of values. In this paper, we show that the observed narrow period range of AXPs is consistent with the accretion model described in CHN if the neutron stars are drawn from an underlying population characterized by broad and reasonable distributions of magnetic field strength, initial spin and accretion disk mass.

It has been suggested that AXPs and SGRs are drawn from the same underlying population of objects. This is motivated by the observation that SGRs (see Hurley 1999 for a review) have properties that are extremely similar to those of AXPs, except for the fact that they occasionally undergo energetic outbursts. We show in this paper that the narrow period range of AXPs and SGRs taken together is also consistent with the above model.

\section{Summary of Possible Neutron Star Spin Histories}

The theoretical model used in this paper is that which is set out in detail in CHN, and to which the reader is referred for details. Here we only summarize the different possible histories of a neutron star accreting from a debris disk.

Such a star will have various spin-down histories depending on the initial values of its free parameters: its surface magnetic field strength $B$, its initial spin period $P_{0}$, and the mass of its accretion disk $M_{d}$. For some values of initial parameters, the star goes into an extended radio pulsar phase in which the magnetospheric radius is greater than the light cylinder radius; accretion action is then assumed to stop. For other initial values, the star is spun down in the propeller phase, during which accreted matter is flung out by centrifugal forces prior to reaching the surface of the star; the star then reaches the quasi-equilibrium "tracking phase", in which the spin of the star roughly matches the equilibrium period but never quite equals it since the mass accretion rate declines with time. If the star enters the tracking phase at a time $t_{\text {trans }}$, then we identify the period between $t_{\text {trans }}$ and the "accretion death time" $2 t_{A D A F}$ to be the AXP phase; here, $t_{A D A F}$ is the time at which the mass accretion rate falls to about $1 \%$ of the Eddington value and the infall becomes an advection-dominated accretion flow or ADAF. For yet other initial values of the star's parameters, it remains in the propeller phase until after the time $2 t_{A D A F}$; in other words, $t_{\text {trans }}>2 t_{A D A F}$. Such a system was called a "propeller system" in CHN.

The X-ray luminosity of the system is determined by the mass accretion rate on to the 
surface of the star, $\dot{M}_{X}$, which could be different from the mass accretion rate through the disk, $\dot{M}$, if some of the material is driven away from the system before reaching the surface of the neutron star. Thus, when accretion action ceases and the star is in the radio pulsar phase, the X-ray luminosity falls to zero. During the propeller phase, most of the matter will be ejected by centrifugal forces, and the system will be X-ray faint, since $\dot{M}_{X} \ll \dot{M}$. During the tracking phase, we assume that $\dot{M}_{X} \sim \dot{M}$ if $\dot{M} \leq \dot{M}_{E}$, where $\dot{M}_{E}$ is the mass accretion rate corresponding to the Eddington luminosity, but that $\dot{M}_{X}=\dot{M}_{E}$ if $\dot{M}>\dot{M}_{E}$; in this phase, the system will be X-ray bright. Note that the assumption here is that even though $\dot{M}$ could be highly super-Eddington, especially at early times, at no point would the X-ray luminosity exceed the Eddington limit, owing to ejection of matter by radiation pressure prior to reaching the surface of the star.

It is not known how rapidly the luminosity will fall during the ADAF phase of the AXP; presumably it will do so faster than linearly in $\dot{M}$; in this paper, for purposes of numerical calculation, we assume the dependence of luminosity between between times $t_{A D A F}$ and

$2 t_{A D A F}$ to be $L_{X} \propto \dot{M}_{X}{ }^{2}$ (of course, for $t<t_{A D A F}, L_{X} \propto \dot{M}_{X}$; and for $t>2 t_{A D A F}, L_{X}=0$ ).

\section{The Distribution of Spin Periods of AXPs}

There are 6 AXPs known today: 1E 2259+586, RXS J170849-4009, 4U 0142+615, 1E 1048-5937, 1E 1841-045 and AXJ 1845-0300 (see Mereghetti 1999). Their spin periods, which are very tightly clustered together, are, in seconds, 6.98, 11.00, 8.69, 6.45, 11.76 and 6.97, respectively. Associated young supernova remnants (SNRs) of estimated age $\sim 10^{4}$ years have so far been detected for 3 of these 6 objects. If it is assumed, as in the model described in the previous section, that all the 6 observed AXPs are the result of supernova explosions $\sim 10^{4}$ years ago, and that the birthrate of radio pulsars in the galaxy is approximately 1 every 100-200 years (see, e.g., Lyne et al. 1998), then the relative birthrates of AXPs and radio pulsars turns out to be $\sim 0.06-0.12$.

How is the distribution of spin periods of the AXPs to be calculated? The evolution in time of the properties of an AXP depends on the three free parameters of the model, namely, the surface magnetic field strength of the neutron star $B_{12}$, the mass of the initial circumstellar accretion disk $M_{d}$, and the initial spin period of the star $P_{0}(\equiv 2 \pi / \Omega(0))$. The way we calculate the distribution of AXP properties is as follows: we specify intrinsic prior probability distributions for each of the parameters $B_{12}, M_{d}$ and $P_{0}$, and endow each neutron star with initial values of $B_{12}, M_{d}$ and $P_{0}$ sampled randomly and independently from their respective distributions, as well as a time of birth chosen randomly from a distribution uniform in time, since we expect the birthrate of neutron stars to be constant 
over time. Having done this for a large number of model neutron stars, we evolve each until a later fiducial time, when we take account of how many stars at that fiducial time are bright AXPs, dead AXPs (i.e., an AXP which is past its $2 t_{A D A F}$ time), propeller systems or radio pulsars. The number of sample neutron stars should be sufficiently large, and the width of their time-of-birth distribution sufficiently long, that the results of this calculation are stable across repetitions of the process.

As an example, we present in detail the results of one such calculation. We take the surface magnetic fields of the neutron stars to be the distribution reported in Narayan \& Ostriker (1990) for radio pulsars: namely, that $\log B$ is distributed normally with a mean of 12.5 and a standard deviation of 0.4 (note that in the rest of this paper, all indicated logarithms are to be taken to base 10). The distributions of $P_{0}$ and $M_{d}$ are poorly constrained by observations. We choose distributions for them that are simple and cover fairly broad ranges of values. $P_{0}$ is chosen to be distributed uniformly between $2 \mathrm{~ms}$ and $50 \mathrm{~ms}$, and $\log \left(M_{d} / M_{\odot}\right)$ is assumed to be distributed uniformly between -6 and -1.8 .

In one particular realization of this calculation, the initial number of neutron stars was selected to be 5,000,000; at the chosen fiducial time, the number of bright AXPs was 139,306; bar graphs showing their distribution of period, luminosity and age are shown in Figure 1. The fractions of all initial neutron stars becoming AXPs, propeller systems and radio pulsars were $0.07,0.10$, and 0.83 , respectively. Thus, the relative birthrates of AXPs and radio pulsars was 0.09 . It will be observed that the AXP spin period distribution peaks at roughly 5-6 seconds, that the ages of the AXPs are of the order of a few times $10^{4}$ years, and that the X-ray luminosity distribution peaks at about $4 \times 10^{35} \mathrm{ergs} / \mathrm{s}$; all of these properties correspond reasonably well with the properties of observed AXPs.

Notice also that the spin period distribution has a long tail towards high periods. However, given that the total number of observed AXPs (whose periods are much more closely clustered) is only 6 , they could still constitute a representative sample from the above distribution. To quantify this statement, we carry out the nonparametric Kolmogorov-Smirnov (K-S) test for two discrete samples (see, e.g., Press et al. 1988) to test whether the hypothetical and observed AXP spin period distributions are compatible.

We define the test statistic to be the quantity $S_{m n} \equiv\left(\frac{m n}{m+n}\right)^{1 / 2} D$, where $m$ and $n$ are the sizes of the observed and hypothetical spin period distributions, respectively (here, $m=6$ and $n=139,306)$, and $D$ is the maximum absolute difference between the cumulative distribution functions of the above distributions. In the above realization of the calculation, $S_{m n}$ has the value 0.76, and it is just significant at the level of significance 0.61 . The cumulative distributions of the observed and hypothetical spin periods are shown in Figure 2 . 
We thus conclude that the observed AXP period distribution is consistent with the hypothetical distribution predicted by our model.

The strongest factor determining the AXP properties in our model is the $B$ distribution; the spin period distribution in Figure 1 can be reasonably well-fitted by a log-normal distribution, reflecting the log-normal distribution of $B$. The weakest factor is the initial spin period $P_{0}$; changing its distribution does not significantly alter the final AXP properties.

The above results are for the case in which $\log \left(M_{d} / M_{\odot}\right)$ is distributed uniformly between -6 and -1.8. The AXPs in this model come from the relatively high end of the $M_{d}$ distribution (in the above realization, the peak value for the AXPs of $\log \left(M_{d} / M_{\odot}\right)$ is -1.8 , and the minimum is at approximately -3.4); neutron stars with smaller disk masses end up as propeller systems or radio pulsars.

One would thus expect that lowering the lower limit of the $\log \left(M_{d} / M_{\odot}\right)$ distribution would not change the AXP properties described above, except that it would lower the ratio of birthrates of AXPs and radio pulsars, since a greater fraction of neutron stars would then have accretion disks too little massive to become AXPs. This is indeed the case: for lower limits of $-5,-6$ and -7 , the corresponding fractions of neutron stars which become AXPs are $0.09,0.07$ and 0.06 ; the fractions of neutron stars becoming X-ray dim propeller systems are $0.14,0.10$ and 0.08 , respectively; the remaining neutron stars become radio pulsars, the fractions of which are $0.77,0.82$ and 0.86 , respectively; hence, the corresponding ratios of birthrates of AXPs and radio pulsars are 0.12, 0.09 and 0.07 .

On the other hand, changing the upper limit of the $\log \left(M_{d} / M_{\odot}\right)$ distribution changes not only the AXP-radio pulsar birthrate ratio, but also the AXP spin distribution: raising it shifts the peak of the period distribution towards lower periods, and lowering it shifts the peak towards higher periods. This affects substantially the level of significance of the K-S test: for upper limits of $-1.7,-1.8$ and -1.9 , the values of $S_{m n}$ are approximately 0.90 , 0.76 and 0.89 respectively, with corresponding levels of significance of $0.39,0.61$ and 0.41 . The relative birthrates of AXPs, propeller systems and radio pulsars also change: the respective fractions of neutron stars becoming AXPs are 0.09, 0.07 and 0.06; the fractions of neutron stars which become propeller systems are $0.11,0.10$ and 0.10 , repsectively; the corresponding fractions of neutron stars which become radio pulsars are $0.81,0.83$ and 0.84 ; hence, the ratios of birthrates of AXPs and radio pulsars for the above three cases are 0.11 , 0.09 and 0.07 , respectively.

It is interesting to apply our model to try to explain the narrow period distribution of the AXPs and SGRs taken together. There are four known SGRs: SGR 1627-41, SGR 
0525-66, SGR 1806-20 and SGR 1900+14; their spin periods are, in seconds, 6.41, 8.0, 7.48 and 5.16 respectively. If we take the $10 \mathrm{AXPs}$ and SGRs together and apply the K-S test to check whether this distribution is consistent with our usual model with $\log \left(M_{d} / M_{\odot}\right)$ distributed uniformly between -6 and -1.8 , we find (with $m=10$, in this case) that $S_{m n}=0.98$, and it is just significant at the level of significance 0.29. Thus, the combined AXP and SGR spin distribution is also consistent with our hypothetical distribution.

\section{Conclusion}

We have shown in this paper that according to our model for fallback accretion disks around solitary neutron stars, not all isolated neutron stars which are the product of supernova explosions become radio pulsars; a substantial fraction of them $(\sim 5-10 \%$, for the models considered here) end up as X-ray bright AXPs, a possibly larger fraction $(\sim 8-14 \%)$ as X-ray faint propeller systems, while the remainder end up as radio pulsars. If the 6 observed AXPs were produced by supernova explosions $\sim 10^{4}$ years ago, then the ratio of birthrates of AXPs and radio pulsars is expected to be of the order of $10 \%$.

Whether a neutron star becomes a radio pulsar, an AXP or a propeller system depends on the values of its surface magnetic field $B$, initial spin period $P_{0}$, and initial circumstellar disk mass $M_{d}$.

Using the observationally constrained distribution function of $B$ for radio pulsars, and assuming simple distribution functions of initial spin period and disk mass covering a broad range in $P_{0}$ and $\log \left(M_{d}\right)$ respectively, it has been shown that our accretion model produces a population of AXPs with a spin period distribution peaking at $\sim 6$ seconds, X-ray luminosity $\sim 10^{35} \mathrm{ergs} / \mathrm{s}$, age $\sim 10^{4}$ years, and with approximately the above AXP-radio pulsar birthrate ratio. Systems with relatively high values of $B$ and $M_{d}$ end up as AXPs, while those with low values end up as radio pulsars; systems with intermediate values of these parameters become propeller systems.

It is difficult to make definitive statements about possible relations between the properties of the underlying neutron star, such as its magnetic field strength or its initial accretion disk mass, and its observable properties such as spin period, age or luminosity, since the wide ranges of values the initial parameters can take give rise to a lot of scatter in the final AXP properties that can be observed. Nevertheless, some rough general trends may be discerned. For example, if a neutron star has a low disk mass ("low" and "high" here are to be understood relatively in the context of the corresponding distributions considered in the previous section and in Figure 1), then it would need a high surface 
magnetic field strength in order to become a visible AXP; this would in turn tend to produce a probable observed spin period which lies slightly rightwards of the peak of the spin period distribution in Figure 1; the probable observed age is low, and the observed luminosity could take a range of values. On the other hand, a star with a high disk mass could reach the AXP phase with a range of surface magnetic field strengths, and the probable observed spin period, luminosity and age could also span a range of values.

In this paper, we wished also to address ourselves to the narrow spin period range observed in AXPs. One might suspect that this would in turn constrain the parameters of any model of AXPs to unphysically slender ranges of values. However, we have shown in this paper that even under the assumption of quite reasonable and broad distributions of the parameters, our model leads to a spin period distribution that is statistically consistent with the narrowly clustered distribution observed in the case of the 6 known AXPs, and also in the case of the 6 AXPs and 4 SGRs taken together (if indeed they can be grouped together as fundamentally the same kind of objects).

We note in conclusion that the above model would not work as a viable explanation for AXPs if the underlying neutron stars had magnetar-like field strengths $\left(B \sim 10^{14.5} \mathrm{G}\right)$; in this case, spin-down would be extremely rapid and would produce spin periods of hundreds of seconds, rather than close to 10 seconds. Thus, it is unnecessary to invoke neutron stars with non-standard magnetic fields in the context of this model for AXPs.

We are particularly grateful to Ramesh Narayan for help and advice during the conception of this paper. We would also like to thank Josh Grindlay and Vicki Kaspi for useful discussion.

\section{REFERENCES}

Alpar, M.A. 1999, astro-ph/9912228

Chatterjee, P., Hernquist, L. \& Narayan, R. 1999, ApJ, in press, astro-ph/9912137 (CHN)

Colpi, M., Geppert, U. \& Page, D. 2000, ApJ, 529, L29

Ghosh, P., Angelini, L. \& White, N.E. 1997, ApJ, 478, 713

Heyl, J.S. \& Hernquist, L. 1997a, ApJ, 491, L95

Heyl, J.S. \& Hernquist, L. 1997b, ApJ, 489, L67

Heyl, J.S. \& Hernquist, L. 1998, MNRAS, 297, L69 
Hulleman, F., van Kerkwijk, M.H., Verbrunt, F.W.M. \& Kulkarni, S.R. 2000, astro$\mathrm{ph} / 0002474$

Hurley, K. 1999, astro-ph/9912061

Kaspi, V. M., Chakrabarty, D. \& Steinberger, J. 1999, ApJ, 525, L33

Kouveliotou, C., Dieters, S., Strohmayer, T., van Paradijs, J., Fishman, G.J., Meegan, C.A., Hurley, K., Kommers, J., Smith, I., Frail, D. \& Murakami, T. 1998, Nature, 393,235

Kouveliotou, C., Strohmayer, T., Hurley, K., van Paradijs, J., Finger, M.H., Dieters, S., Woods, P., Thompson, C. \& Duncan, R.C. 1999, ApJ, 510, L115

Lyne, A.G., Manchester, R.N., Lorimer, D.R., Bailes, M., D’Amico, N., Tauris, T.M., Johnston, S., Bell, J.F., Nicastro, L. 1998, MNRAS, 295, 743L

Marsden, D., Rothschild, R.E. \& Lingenfelter, R.E. 1999, ApJ, 520, L107

Marsden, D., Lingenfelter, R.E., Rothschild, R.E. \& Higdon, J.C. 1999, astro-ph/9912207

Mereghetti, S. 1999, astro-ph/9911252

Mereghetti, S. \& Stella, L. 1995, ApJ, 442, L17

Mereghetti, S., Israel, G.L. \& Stella, L. 1998, MNRAS, 296, 689

Narayan, R. \& Ostriker, J.P. 1990, ApJ, 352, 222

Perna, R., Hernquist, L. \& Narayan, R. 1999, astro-ph/9912297

Press, W.H., Flannery, B.P., Teukolsky, S.A. \& Vetterling, W.T. 1988, Numerical Recipes in $\mathrm{C}$ (Cambridge: Cambridge Univ. Press)

Thompson, C. \& Duncan, R.C. 1996, ApJ, 473, 322

Van Paradijs, J., Taam, R.E. \& van den Heuvel, E.P.J. 1995, A\&A, 299, 41

Wang, J.C.L. 1997, ApJ, 486, L119

Wilson, C.A., Dieters, S., Finger, M.H., Scott, D.M. \& van Paradijs, J. 1999, ApJ, 513, 464 

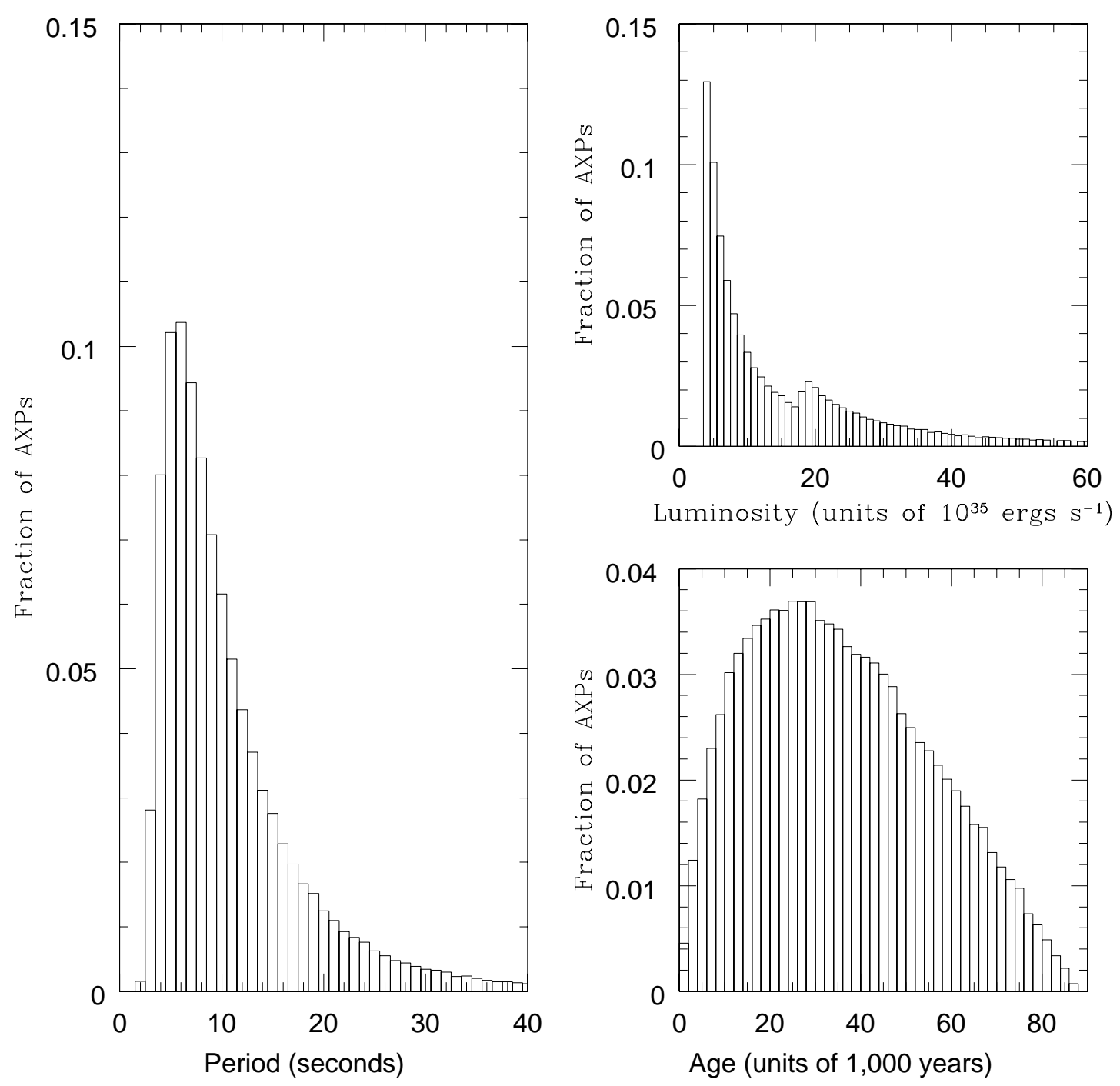

Fig. 1.- Shown are the distributions of AXP spin period, X-ray luminosity and age for a model in which $\log B$ is distributed normally with a mean of 12.5 and a standard deviation of $0.4, P_{0}$ is distributed uniformly between $2 \mathrm{~ms}$ and $50 \mathrm{~ms}$, and $\log \left(M_{d} / M_{\odot}\right)$ is distributed uniformly between -6 and -1.8 . Note that the luminosity distribution has two peaks; this is due to the fact that we have chosen a prescription for the X-ray luminosity that is continuous though not smooth at the time $t=t_{A D A F}: L_{X} \propto \dot{M}_{X}$ for $t<t_{A D A F}$ and $L_{X} \propto \dot{M}_{X}{ }^{2}$ for $t_{A D A F} \leq t \leq 2 t_{A D A F}$. An AXP is most likely to lie near the primary peak, which corresponds to low luminosity systems in the $t_{A D A F} \leq t \leq 2 t_{A D A F}$ regime, while the region of the distribution rightwards of the primary peak corresponds to higher luminosity AXPs in the $t<t_{A D A F}$ regime. Note also that for the parameters of the neutron star chosen in this paper, the Eddington luminosity $L_{E}=1.8 \times 10^{38} \mathrm{ergs} \mathrm{s}^{-1}$. 


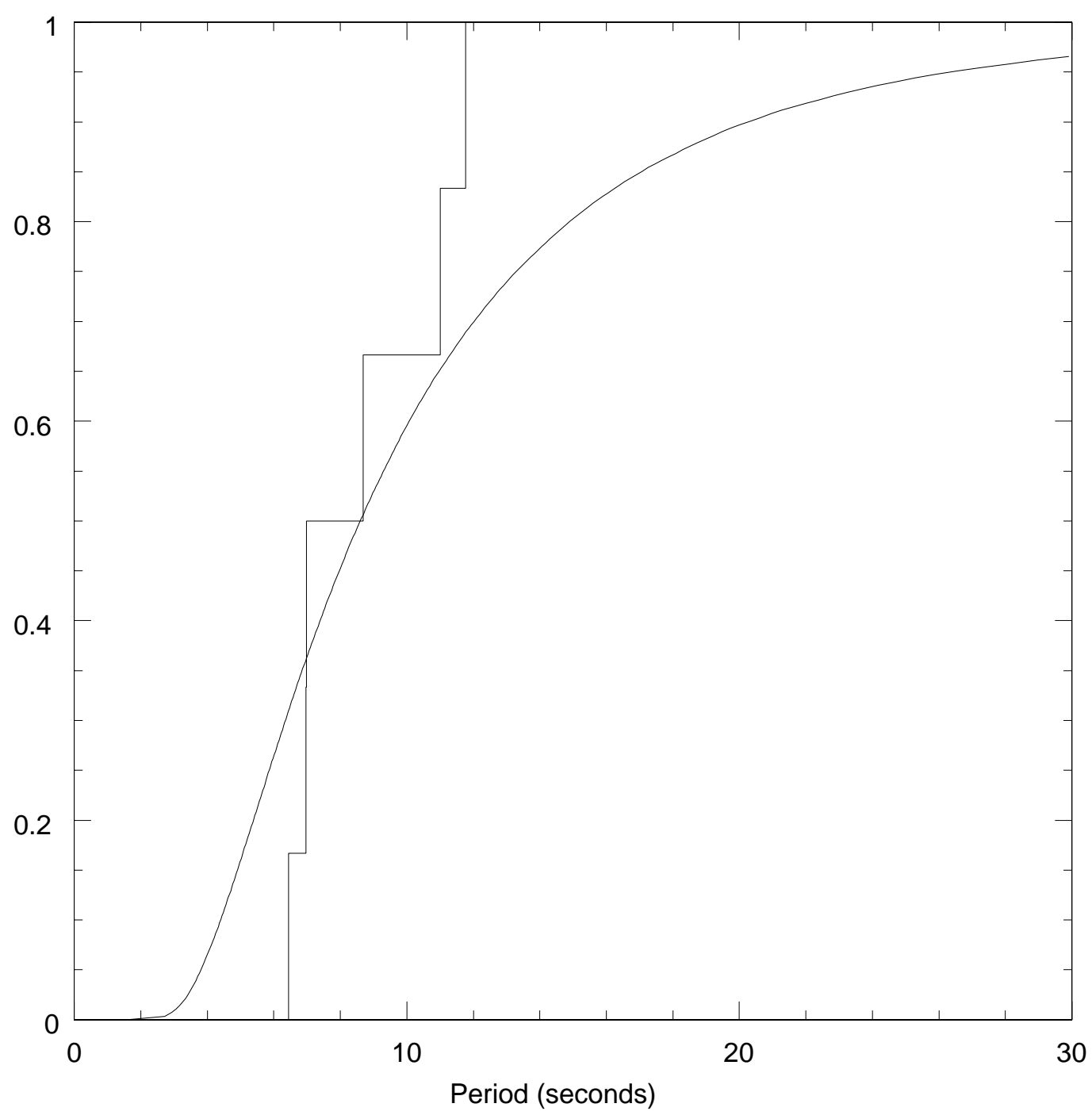

Fig. 2.- The smooth curve is the cumulative probability distribution of the theoretical AXP spin-period distribution for a model in which $\log B$ is distributed normally with a mean of 12.5 and a standard deviation of $0.4, P_{0}$ is distributed uniformly between 2 ms and $50 \mathrm{~ms}$, and $\log \left(M_{d} / M_{\odot}\right)$ is distributed uniformly between -6 and -1.8 . The step-function is the cumulative distribution of the 6 known AXPs. The maximum distance between the two curves is $D=0.31$ at a spin period of 11.76 seconds. This value is just significant at a Kolmogorov-Smirnov-test level of significance of 0.61 . 